\title{
The Pediatric Index of Mortality as a Trigger Tool for the Detection of Serious Errors and Adverse Events
}

\author{
Rüegger, Christoph M ; Frey, Bernhard
}

\begin{abstract}
OBJECTIVES To test the hypothesis that patients who die in a PICU despite a low predicted mortality at PICU admission are affected by serious errors and adverse events. DESIGN Retrospective cross-sectional review of medical records for serious errors and adverse events. SETTING Tertiary interdisciplinary neonatal PICU. PATIENTS All admissions to our PICU who died despite a low expected mortality (Pediatric Index of Mortality) of less than 10\% (trigger-positive admissions). They were compared with a random sample of 100 PICU admissions with a Pediatric Index of Mortality of less than $10 \%$ who survived (trigger-negative admissions). INTERVENTIONS None. MEASUREMENTS AND MAIN RESULTS There were 7,383 admissions (91\%) with a Pediatric Index of Mortality 2 below 10\%. Seventytwo trigger-positive admissions and 100 trigger-negative admissions met the criteria for detailed chart review. Forty-five serious errors and adverse events were identified, 0.47 per trigger-positive admission and 0.11 per trigger-negative admission $(\mathrm{p}<0.001)$. Nineteen serious errors and adverse events $(42 \%)$ were related to clinical sepsis acquired during the PICU stay, 17 (89\%) in trigger-positive admissions and two $(11 \%)$ in trigger-negative admissions $(\mathrm{p}<0.001)$. A further 18 serious errors and adverse events $(40 \%)$ were intervention related, nine (50\%) in trigger-positive admissions and nine $(50 \%)$ in trigger-negative admissions $(\mathrm{p}=0.46)$. Eight serious errors and adverse events $(18 \%)$ were associated with medication use, all of which occurred in trigger-positive admissions $(\mathrm{p}=0.001)$. The median (interquartile range) age for admissions with and without serious errors and adverse events was 0.3 months (0.0-4.6 mo) and 7.4 months (0.4-58.4 mo) ( $\mathrm{p}<0.001)$, and their median (interquartile range) duration of invasive ventilation was 140 hours $(50-451 \mathrm{hr})$ and 2 hours $(0-41 \mathrm{hr})(\mathrm{p}<0.001)$, respectively. CONCLUSIONS The records of PICU patients with a low expected mortality at admission and death in PICU should be reviewed routinely and/or discussed at morbidity and mortality meetings. These patients may have experienced more in-hospital safety-related events compared with PICU patients with a low Pediatric Index of Mortality who survived. Such adverse events may be amenable to system changes, thus improving patient care.
\end{abstract}

DOI: https://doi.org/10.1097/PCC.0000000000001654

Posted at the Zurich Open Repository and Archive, University of Zurich

ZORA URL: https://doi.org/10.5167/uzh-161831

Journal Article

Published Version

Originally published at:

Rüegger, Christoph M; Frey, Bernhard (2018). The Pediatric Index of Mortality as a Trigger Tool for the Detection of Serious Errors and Adverse Events. Pediatric Critical Care Medicine, 19(9):869-874.

DOI: https://doi.org/10.1097/PCC.0000000000001654 


\title{
The Pediatric Index of Mortality as a Trigger Tool for the Detection of Serious Errors and Adverse Events*
}

\author{
Christoph M. Rüegger, $\mathrm{MD}^{1,2}$; Bernhard Frey, $\mathrm{MD}^{1}$
}

\begin{abstract}
Objectives: To test the hypothesis that patients who die in a PICU despite a low predicted mortality at PICU admission are affected by serious errors and adverse events.
\end{abstract}

Design: Retrospective cross-sectional review of medical records for serious errors and adverse events.

Setting: Tertiary interdisciplinary neonatal PICU.

Patients: All admissions to our PICU who died despite a low expected mortality (Pediatric Index of Mortality) of less than 10\% (trigger-positive admissions). They were compared with a random sample of 100 PICU admissions with a Pediatric Index of Mortality of less than 10\% who survived (trigger-negative admissions).

Interventions: None.

Measurements and Main Results: There were 7,383 admissions (91\%) with a Pediatric Index of Mortality 2 below 10\%. Seventytwo trigger-positive admissions and 100 trigger-negative admissions met the criteria for detailed chart review. Forty-five serious errors and adverse events were identified, 0.47 per trigger-positive admission and 0.11 per trigger-negative admission $(p<$ $0.001)$. Nineteen serious errors and adverse events (42\%) were related to clinical sepsis acquired during the PICU stay, 17 (89\%) in trigger-positive admissions and two (11\%) in trigger-negative admissions $(p<0.001)$. A further 18 serious errors and adverse events (40\%) were intervention related, nine (50\%) in trigger-positive admissions and nine $(50 \%)$ in trigger-negative admissions $(p=0.46)$. Eight serious errors and adverse events $(18 \%)$ were associated with medication use, all of which occurred in triggerpositive admissions ( $p=0.001$ ). The median (interquartile range) age for admissions with and without serious errors and adverse

\footnotetext{
*See also p. 903.

'Department of Intensive Care and Neonatology, University Children's Hospital Zürich, Switzerland.

${ }^{2}$ Newborn Research, Department of Neonatology, University Hospital and University of Zürich, Zürich, Switzerland.

Supported, in part, by Early Postdoc.Mobility fellowship of the Swiss National Science Foundation (P2ZHP3_161749) (to Dr. Rüegger).

The authors have disclosed that they do not have any potential conflicts of interest.

For information regarding this article, E-mail: christoph.rueegger@usz.ch

Copyright (c) 2018 by the Society of Critical Care Medicine and the World Federation of Pediatric Intensive and Critical Care Societies

DOI: 10.1097/PCC.0000000000001654
}

events was 0.3 months (0.0-4.6 mo) and 7.4 months (0.4-58.4 mo) $(p<0.001)$, and their median (interquartile range) duration of invasive ventilation was 140 hours $(50-451 \mathrm{hr})$ and 2 hours (0-41 hr) $(p<0.001)$, respectively.

Conclusions: The records of PICU patients with a low expected mortality at admission and death in PICU should be reviewed routinely and/or discussed at morbidity and mortality meetings. These patients may have experienced more in-hospital safety-related events compared with PICU patients with a low Pediatric Index of Mortality who survived. Such adverse events may be amenable to system changes, thus improving patient care. (Pediatr Crit Care Med 2018; 19:869-874)

Key Words: adverse drug event; adverse event; patient safety; pediatric Index of Mortality; pediatric intensive care unit; trigger

$\mathrm{H}$ ospitalized children are vulnerable to safety-related events, particularly those in high-acuity environments such as PICUs (1). The majority of methods that have been used thus far to identify serious errors and adverse events (SEAEs) are based on chart reviews, voluntary reporting by healthcare providers, direct observations, and reviews of medical malpractice claims (2-4). A different strategy known as "the trigger tool methodology" has emerged as a practical, reliable, and effective means for the identification of SEAEs in hospitalized children (5-10). A trigger is a predefined clinical event identified by medical record review that "triggers" further investigations to determine the presence or absence of an adverse event (AE) (11).

The Pediatric Index of Mortality (PIM) is a simple, reliable, and widespread mortality prediction model; the data for this model are collected at the time of first contact of the patient with the PICU. The PIM was developed in 1996 (12), updated in 2003 (PIM2) (13) and 2013 (PIM3) (14), and has become an important model for monitoring and comparing the risk-adjusted expected mortality between and within ICUs over time.

Children who die in PICU despite a low predicted mortality at admission may have suffered a SEAE, potentially contributing to death. Therefore, in addition to other AE identification methods, the case histories of these patients should be analyzed. In this study, we want to test the hypothesis that patients 
who die in PICU despite a low predicted mortality reveal a higher number of SEAEs than patients with a low predicted mortality who survive. We sought to describe the occurrence of SEAEs detected by this method and analyze the SEAEs and the patients involved.

\section{MATERIALS AND METHODS}

This was a retrospective cross-sectional study conducted in a tertiary, interdisciplinary PICU (18 beds). The unit includes postcardiac surgery patients and runs an extracorporeal membrane oxygenation program. Approximately $25 \%$ of the patients are in the neonatal age group, and these patients are primarily neonates with cardiac and/or surgical pathologies. The following demographic and illness severity data for all patients who were admitted to our PICU between January 1, 2007, and August 31, 2013, were prospectively recorded in our hospital information system at the time of admission (i.e., the minimal dataset of the Swiss Society of Intensive Care) (15): age, length of stay (LOS) in the PICU, expected mortality at admission (PIM2), artificial ventilation (invasive and noninvasive), and diagnosis (main diagnosis at admission according to the Australian and New Zealand Pediatric Intensive Care Registry) (16).

Our study group consisted of patients with a PIM2 of less than $10 \%$ at first contact with PICU, who died during their intensive care stay (trigger-positive admissions [TPAs]). The PIM2 cutoff of $10 \%$ was chosen because it combines a reasonably low expected mortality with a high number of patients (in our PICU around 90\% of admissions fall into this PIM2 stratum). Of all patients with a PIM2 score of less than $10 \%$ who survived, a random sample of 100 patients was selected using an online random number generator (17). This sample constituted our control group (trigger-negative admissions [TNAs]). Admissions with incomplete medical records resulting in missing PIM data were excluded.

The medical records of the TPAs and TNAs were reviewed by the first author of this article ( $5 \mathrm{yr}$ of clinical experience in neonatal and pediatric critical care) for serious medical errors and serious AEs. These were defined by Wilson et al (18) as unintended injuries or complications that result in disability, death, or prolonged hospital stay and that are caused by healthcare management rather than the disease process. All entries made into the medical records of the included admissions were screened for severe iatrogenic incidents leading to hemodynamic or respiratory instability and included nosocomial septic shock, intervention-related complications, and serious adverse drug events (SADEs). We used the previously published definition for septic shock requiring the presence of systemic inflammatory response syndrome with cardiovascular organ dysfunction in the context of a suspected or proven infection (19). SADEs were defined as serious, harmful, and unintended consequences of medication use (20).

We used frequencies and percentages to present categorical data. Medians and interquartile ranges (IQRs) were given for continuous data. A significant difference was defined as a $p$ value of less than 0.05 using the appropriate statistical test for categorical $\left(\chi^{2}\right)$ or continuous (Mann-Whitney $U$ ) data. All analyses were performed using STATA software (Intercooled V.14; StataCorp, College Station, TX). Ethics committee approval was obtained for the analysis of the database.

\section{RESULTS}

During the study period, there were 8,116 admissions to our PICU. Twenty-five admissions had incomplete medical records at the time of entry, which left 8,091 admissions for further analysis. Their observed mortality was 2.7\% (221 deaths per 8,091 admissions). Their median (IQR) PIM2 at first contact with PICU was $1.5 \%(0.75-3.27 \%)$.

There were 7,383 admissions (91.2\%) with a PIM2 of less than $10 \%$. Seventy-two TPAs and 100 randomly selected TNAs met the criteria for detailed chart review. Their group characteristics are given in Table 1. The leading causes of death among the patients who died with low PIM were cardiac disorders (32\%), including cardiac failure and arrhythmia, respiratory failure $(26 \%)$, brain death (due to intracranial hemorrhage, intracranial hypertension, or hypoxic ischemic encephalopathy) (15\%), septic shock (14\%), intractable pulmonary hypertension (7\%), and miscellaneous causes (6\%).

Among the 172 TPAs and TNAs, 39 admissions (23\%) were affected by SEAEs. Overall, 45 SEAEs were identified $(0.26$ SEAE per admission), 34 in TPAs (0.47 SEAE per TPA) and 11 in TNAs $(0.11$ SEAE per TNA) $(p<0.001)$.

Nineteen SEAEs (42\%) were associated with nosocomial bloodstream infections and clinical sepsis acquired during the PICU stay, $17(89 \%)$ in the trigger-positive and two (11\%) in the trigger-negative group $(p<0.001)$. Blood cultures were positive in 13 admissions. Positive blood cultures showed coagulase-negative Staphylococci $(n=5)$, Escherichia coli $(n=2)$, Staphylococcus aureus $(n=2)$, Klebsiella pneumoniae $(n=2)$, Pseudomonas aeruginosa $(n=1)$, and Acinetobacter baumanii $(n=1)$. Eighteen SEAEs $(40 \%)$ were intervention related, nine $(50 \%)$ in TPAs and nine in TNAs $(50 \%)(p=0.31)$. The different types of interventions and their related SEAEs are provided in Table 2. Eight SEAEs (18\%) were associated with medication use: sedation $(n=3)$, anticoagulation $(n=2)$, catecholamines $(n=2)$, and intoxication $(n=1)$. All SADEs occurred in TPAs $(p<0.001)$.

Table 3 compares the admissions with and without SEAEs. The median age (IQR) was 0.3 months $(0.0-4.6 \mathrm{mo})$ for admissions with SEAEs and 7.4 months (0.4-58.4 mo) for admissions without SEAEs $(p<0.001)$. The median (IQR) length of invasive ventilation was 140 hours $(50-451 \mathrm{hr})$ in the SEAEpositive group and 2 hours $(0-41 \mathrm{hr})$ in the SEAE-negative group $(p<0.001)$.

\section{DISCUSSION}

The purpose of this study was to test the use of the PIM score as a PICU-specific, simple, and efficient trigger tool for the identification of SEAEs. We hypothesized that patients who die in the PICU despite a low predicted mortality (PIM2 < 10\%) 


\section{TABLE 1. Group Characteristics of Trigger-Positive and Trigger-Negative Admissions}

\begin{tabular}{|c|c|c|c|}
\hline Group Characteristics & $\begin{array}{c}\text { Trigger-Positive } \\
\text { Admissions }(n=72)\end{array}$ & $\begin{array}{c}\text { Trigger-Negative } \\
\text { Admissions }(n=100)\end{array}$ & p \\
\hline Male, $n(\%)$ & $38(53)$ & $65(65)$ & 0.11 \\
\hline Age, mo, median (IQR) & $0.3(0.0-5.6)$ & $21.5(2.1-97.6)$ & $<0.001$ \\
\hline Pediatric Index of Mortality II (\%), median (IOR) & $5.1(2.3-7.2)$ & $1.1(0.6-2.0)$ & $<0.001$ \\
\hline Postprocedural ${ }^{\mathrm{a}}$, delivery room & $23(32)$ & $57(57)$ & 0.001 \\
\hline Emergency department & $7(10)$ & $11(11)$ & 0.79 \\
\hline Ward & $24(33)$ & $18(18)$ & 0.02 \\
\hline Invasive ventilation, $n(\%)$ & $62(86)$ & $45(45)$ & $<0.001$ \\
\hline Length of invasive ventilation (hr), median (IQR) & $94(13-398)$ & $0(0-13)$ & $<0.001$ \\
\hline Noninvasive ventilation, $n(\%)$ & $19(26)$ & $3(3)$ & $<0.001$ \\
\hline Length of noninvasive ventilation (hr), median (IOR) & $0(0-1)$ & $0(0-0)$ & $<0.001$ \\
\hline Length of stay in PICU (d), median (IOR) & $11(2-22)$ & $1(1-3)$ & $<0.001$ \\
\hline
\end{tabular}

IQR $=$ interquartile range.

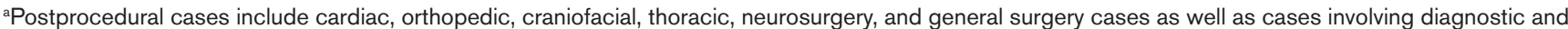
interventional cardiac catheters.

${ }^{\text {b}}$ Others include ambulance and air rescue service cases.

\section{TABLE 2. Serious Errors and Adverse Events in the Context of Medical Interventions}

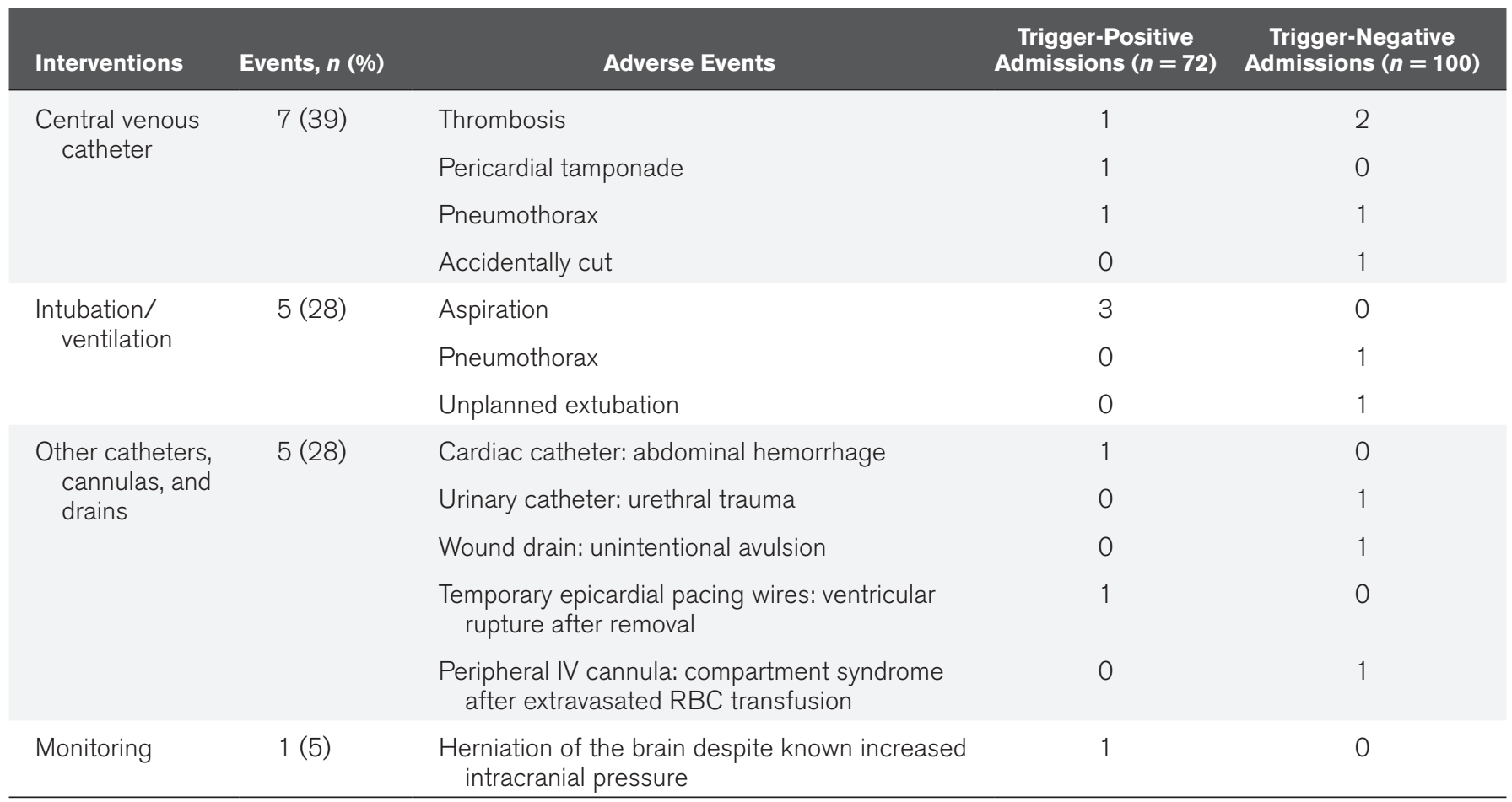




\section{TABLE 3. Group Characteristics of Admissions With and Without Serious Errors and} Adverse Events

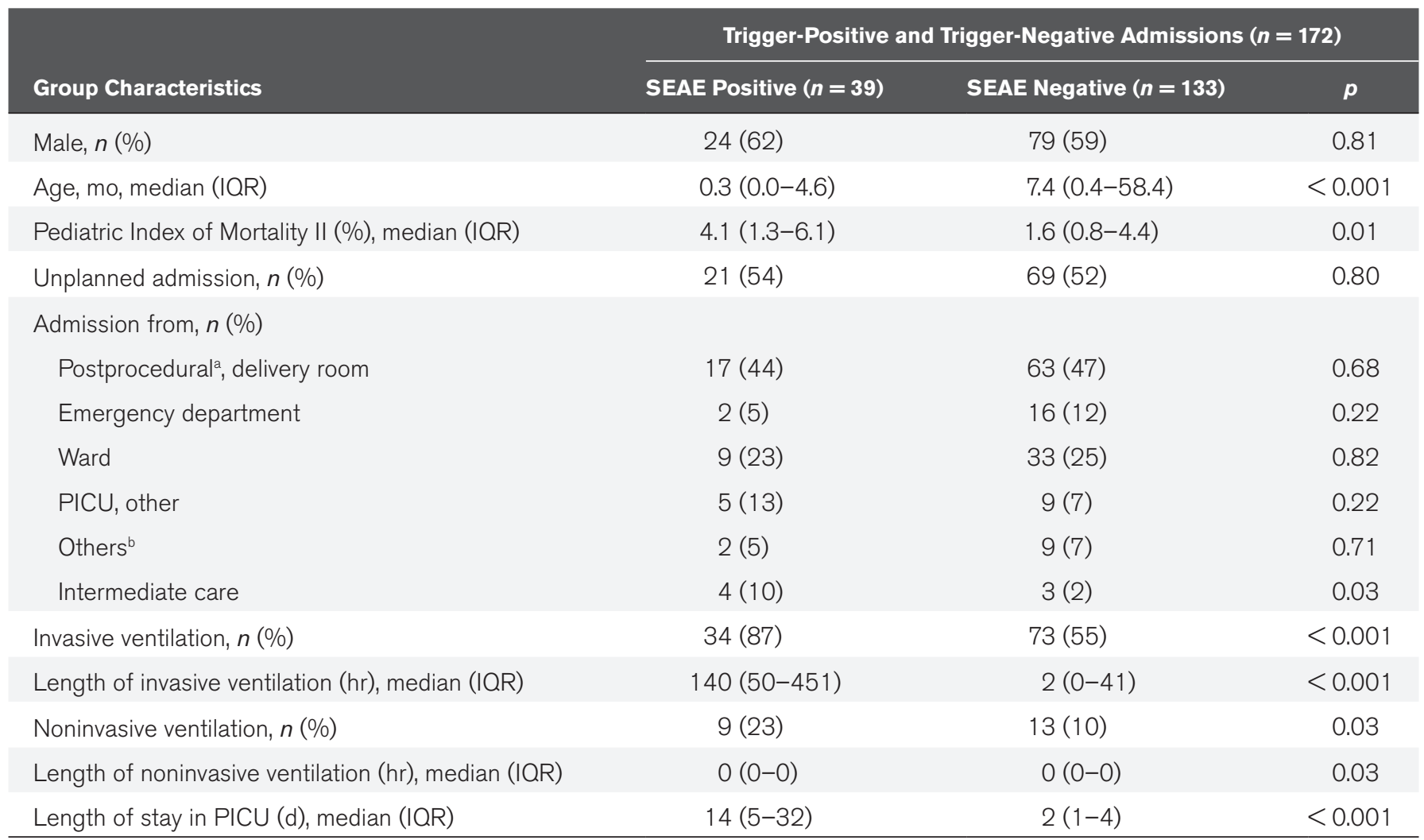

$\mathrm{IQR}=$ interquartile ranges, $\mathrm{SEAE}=$ serious errors and adverse event.

aPostprocedural cases include cardiac, orthopedic, craniofacial, thoracic, neurosurgery, and general surgery cases as well as cases involving diagnostic and interventional cardiac catheters.

${ }^{b}$ Others include ambulance and air rescue service cases.

reveal a higher number of SEAEs than patients with a low predicted mortality who survive. Our trigger tool allowed us to detect 0.26 SEAEs per admission, 0.47 per TPA and 0.11 per TNA $(p<0.001)$. The majority of SEAEs were related to nosocomial clinical sepsis and medical interventions. SADEs were less frequent; however, their occurrence was restricted to TPAs. Younger patients and patients with prolonged ventilator dependence were at higher risks of SEAEs. This finding is in accordance with the literature evaluating safety incidents in the PICU $(21,22)$.

Previous studies have established harm rates of 0.37 (9) and 0.40 (7) per admission using trigger lists to identify the most common causes of harm in pediatric inpatient environments. Our SEAE rate of 0.47 per TPA is slightly higher, even though structured chart reviews were only performed for the patients selected by our trigger method. Furthermore, we concentrated on severe AEs (SEAEs) only. This might indicate a reliable selection of critically ill children at risk of in-hospital safetyrelated events with our PIM-based trigger tool. Agarwal et al Agarwal reported an $\mathrm{AE}$ and adverse drug event (ADE) rate of 2.02 per patient as identified with a PICU trigger tool that involved the use of an instruction manual containing detailed definitions of triggers, possible associated predefined AEs, and case examples (10). Their higher AE rate may be attributable to the fact that they also screened for minor AEs, whereas we did not.

Half of the SEAEs among TPAs were related to septic shock that occurred after admission to the PICU. Previous research demonstrated that the majority of these cases are associated with intravascular catheter placement (23). This finding is consistent with our observation that half of the TPAs with SEAEs were admitted from theatre, where the placement of central catheters is routine. This observation is important because central line-associated bloodstream infections (CLABSI) are coupled with higher attributable morbidity and mortality rates $(24,25)$. In addition to the increased risk of CLABSI due to multiple catheters, postoperative patients are likely to be sedated. This care can have a profound effect on respiratory effort and drive. Consequently, medical devices, such as ventilators, are necessary in the care of critically ill surgical patients. However, these tools may also be associated with increased risks due to human errors $(26,27)$. Indeed, our data indicate an association between the occurrence of SEAEs and the length of invasive ventilation (Table 3). Assuming that an increase in the risk of SEAEs is related to medical devices, one could expect that mechanical ventilation, particularly when prolonged, would be associated with the occurrence of SEAEs and not the reverse. Furthermore, this association emphasizes 
that surgical patients are a high-risk population within the PICU that deserves special attention for SEAE reduction.

We found eight SEAEs in the context of medication use. Previous reports about ADEs in PICUs suggested that the use of multiple drugs as well as lesser patient age may favor the occurrence of $\operatorname{ADEs}(28,29)$. This is consistent with our observation that all SADEs occurred in the group of TPAs. This group of infants was significantly younger and sicker compared with infants in the TNAs group, as shown in Table 1.

Additional risk factors for poor outcomes found in our study were unplanned admission to PICU and admission from the wards (Table 1): Two thirds of the TPAs to the PICU were unplanned, and a third was transferred from the study hospital's wards. Prior studies have reported similar findings; Odetola et al (30) found that, compared with admissions from the emergency department, patients admitted from the wards exhibit increased illness severities, longer PICU LOS, and greater odds of mortality. Unplanned admission from the ward to the PICU might additionally be a marker of poor response or nonresponse to therapy that results in a poor outcome. These poor or failed responses might partially be explained by a delayed transfer to the PICU and the consequent progression of the original condition that prompted the initial hospitalization (31).

Our trigger tool may enhance the discovery of AEs and may be an excellent supplement to other AE identification methods, such as mortality and morbidity meetings (32). However, mortality and morbidity meetings usually focus on the severity of illness toward the end of life, whereas the initial admission factors are less visible. Hence, all PICU deaths should be discussed in morbidity and mortality meetings, especially deaths that occurred despite low illness severity at PICU admission. These deaths may be preventable, given their low predicted mortality.

It is questionable whether another mortality prediction model, such as the Pediatric Risk of Mortality (PRISM) score, would be better suited for detecting harm (33). In contrast to the PIM score, which includes the data acquired at admission, the PRISM III is calculated from the most abnormal variables that are observed during the first 24 hours of the PICU stay. Therefore, the PRISM is influenced by PICU treatment; that is, a child who is mismanaged or suffers a serious $\mathrm{AE}$ within the first 24 hours in the PICU will be given a score that suggests severe illness. For this reason, the PRISM may be less suitable as a trigger tool. Additional research comparing PIM2- and PRISM III-based trigger tools in the detection of SEAEs is needed to resolve this question.

Our study has several limitations. First, repeated and independent analyses and validations of the medical records by different pediatricians (interrater reliability testing) were not performed. Second, there are some children who present with stable conditions upon PICU admission (i.e., low PIM2 scores) who nonetheless have life-threatening conditions and later die in the PICU (e.g., a newborn admitted to the PICU after birth with hypoplastic left heart syndrome or a child scheduled for major surgery who is preoperatively admitted for logistic reasons). These children may die due to factors that are related to their underlying conditions and are unrelated to SEAEs, and a low PIM2 is less suitable as trigger tool for such children. In our study, seven admissions in the TPA group fell into this category. However, the exclusion of these patients would not substantially change the performance of our trigger tool method (0.48 SEAE/TPA with the patients excluded, 0.47 SEAE/TPA with the patients included). Finally, another limitation is the study's retrospective and single-center design.

\section{CONCLUSIONS}

Our trigger tool (i.e., death in the PICU despite a low PIM at admission) reliably and quickly identified PICU admissions at risk of SEAEs. We found that younger patients and patients with prolonged ventilator dependence were most affected by SEAEs. We identified a considerable number of cases with septic shock acquired in PICU, indicating the high burden of nosocomial infection, particularly among TPAs. Surgical patients, unplanned admissions to PICU, and admissions from the wards were additional high-risk populations within our PICU that deserve special attention for SEAE reduction. By means of focused record reviews or morbidity and mortality meetings, special attention should be put on deaths with low predicted mortality. This may enhance the discovery of SEAEs. They may be amenable to system changes and may therefore result in improved patient safety.

\section{REFERENCES}

1. Beal AC, Co JP, Dougherty D, et al: Quality measures for children's health care. Pediatrics 2004; 113(1 Pt 2):199-209

2. Woods D, Thomas E, Holl J, et al: Adverse events and preventable adverse events in children. Pediatrics 2005; 115:155-160

3. Kaushal R, Bates DW, Landrigan C, et al: Medication errors and adverse drug events in pediatric inpatients. JAMA 2001; 285:2114-2120

4. Tibby SM, Correa-West J, Durward A, et al: Adverse events in a paediatric intensive care unit: Relationship to workload, skill mix and staff supervision. Intensive Care Med 2004; 30:1160-1166

5. Miller MR, Elixhauser A, Zhan C: Patient safety events during pediatric hospitalizations. Pediatrics 2003; 111 (6 Pt 1):1358-1366

6. Takata GS, Mason W, Taketomo C, et al: Development, testing, and findings of a pediatric-focused trigger tool to identify medication-related harm in US children's hospitals. Pediatrics 2008; 121:e927-e935

7. Stockwell DC, Bisarya H, Classen DC, et al: A trigger tool to detect harm in pediatric inpatient settings. Pediatrics 2015; 135:1036-1042

8. Hooper AJ, Tibballs J: Comparison of a Trigger Tool and voluntary reporting to identify adverse events in a paediatric intensive care unit. Anaesth Intensive Care 2014; 42:199-206

9. Kirkendall ES, Kloppenborg E, Papp J, et al: Measuring adverse events and levels of harm in pediatric inpatients with the Global Trigger Tool. Pediatrics 2012; 130:e1206-e1214

10. Agarwal S, Classen D, Larsen G, et al: Prevalence of adverse events in pediatric intensive care units in the United States. Pediatr Crit Care Med 2010; 11:568-578

11. Sharek PJ, Horbar JD, Mason W, et al: Adverse events in the neonatal intensive care unit: Development, testing, and findings of an NICU-focused trigger tool to identify harm in North American NICUs. Pediatrics 2006; 118:1332-1340

12. Shann F, Pearson G, Slater A, et al: Paediatric index of mortality (PIM): A mortality prediction model for children in intensive care. Intensive Care Med 1997; 23:201-207 
13. Slater A, Shann F, Pearson G; Paediatric Index of Mortality (PIM) Study Group: PIM2: A revised version of the Paediatric Index of Mortality. Intensive Care Med 2003; 29:278-285

14. Straney L, Clements A, Parslow RC, et al; ANZICS Paediatric Study Group and the Paediatric Intensive Care Audit Network: Paediatric index of mortality 3: An updated model for predicting mortality in pediatric intensive care*. Pediatr Crit Care Med 2013; 14:673-681

15. Goldhill DR, McNarry AF, Hadjianastassiou VG, et al: The longer patients are in hospital before intensive care admission the higher their mortality. Intensive Care Med 2004; 30:1908-1913

16. Slater A, Shann F, McEniery J; ANICS Study Group: The ANZPIC registry diagnostic codes: A system for coding reasons for admitting children to intensive care. Intensive Care Med 2003; 29:271-277

17. Random Number Generator. Available at: https://rechneronline.de/ random-numbers/. Accessed August 1, 2017

18. Wilson RM, Runciman WB, Gibberd RW, et al: The quality in Australian health care study. Med J Aust 1995; 163:458-471

19. Goldstein B, Giroir B, Randolph A: International pediatric sepsis consensus conference: Definitions for sepsis and organ dysfunction in pediatrics. Pediatr Crit Care Med 2005; 6:2-8

20. Bailey $C$, Peddie D, Wickham ME, et al: Adverse drug event reporting systems: A systematic review. Br J Clin Pharmacol 2016; 82:17-29

21. Skapik JL, Pronovost PJ, Miller MR, et al: Pediatric safety incidents from an intensive care reporting system. J Patient Saf 2009; 5:95-101

22. Niesse OW, Sennhauser FH, Frey B: Critical incidents in paediatric critical care: Who is at risk? Eur J Pediatr 2011; 170:193-198

23. Renaud B, Brun-Buisson C; ICU-Bacteremia Study Group: Outcomes of primary and catheter-related bacteremia. A cohort and case-control study in critically ill patients. Am J Respir Crit Care Med 2001; 163:1584-1590
24. Pittet D, Tarara D, Wenzel RP: Nosocomial bloodstream infection in critically ill patients. Excess length of stay, extra costs, and attributable mortality. JAMA 1994; 271:1598-1601

25. Nowak JE, Brilli RJ, Lake MR, et al: Reducing catheter-associated bloodstream infections in the pediatric intensive care unit: Business case for quality improvement. Pediatr Crit Care Med 2010; 11:579-587

26. Larsen GY, Donaldson AE, Parker HB, et al: Preventable harm occurring to critically ill children. Pediatr Crit Care Med 2007; 8:331-336

27. Samore $M H$, Evans RS, Lassen A, et al: Surveillance of medical device-related hazards and adverse events in hospitalized patients. JAMA 2004; 291:325-334

28. Silva DC, Araujo OR, Arduini RG, et al: Adverse drug events in a paediatric intensive care unit: A prospective cohort. BMJ Open 2013; 3:e001868

29. Impicciatore $P$, Choonara I, Clarkson A, et al: Incidence of adverse drug reactions in paediatric in/out-patients: A systematic review and meta-analysis of prospective studies. Br J Clin Pharmacol 2001; 52:77-83

30. Odetola FO, Rosenberg AL, Davis MM, et al: Do outcomes vary according to the source of admission to the pediatric intensive care unit? Pediatr Crit Care Med 2008; 9:20-25

31. Rapoport J, Teres D, Lemeshow S, et al: Timing of intensive care unit admission in relation to ICU outcome. Crit Care Med 1990; 18:1231-1235

32. Frey B, Doell C, Klauwer D, et al: The morbidity and mortality conference in pediatric intensive care as a means for improving patient safety. Pediatr Crit Care Med 2016; 17:67-72

33. Pollack MM, Ruttimann UE, Getson PR: Pediatric risk of mortality (PRISM) score. Crit Care Med 1988; 16:1110-1116 